\title{
PIECEWISE POLYNOMIAL FUNCTIONS, CONVEX POLYTOPES AND ENUMERATIVE GEOMETRY
}

\author{
MICHEL BRION \\ École Normale Supérieure de Lyon \\ 46 allée d'Italie, 69364 Lyon Cedex 7, France \\ E-mail: mbrion@fourier.grenet.fr
}

\section{Introduction.}

This paper explores some of the connections between the objects of its title. It is based on a new approach to McMullen's polytope algebra, and on its relation with equivariant cohomology of toric varieties. In particular, we give another proof of a recent result of Fulton and Sturmfels, which identifies the polytope algebra with the direct limit of all Chow rings of smooth, complete torus embeddings (see [14]). On the other hand, we obtain a version of the classical theorem of Bézout, which holds in any spherical homogeneous space. This generalizes a theorem of Bernstein and Kouchnirenko: The number of common points to $d$ hypersurfaces in general position in a $d$-dimensional torus is $d$ ! times the mixed volume of the associated Newton polytopes (see [2], [18] and also [13] 5.5).

Given a finite-dimensional vector space $V$ over an ordered field $K$, there is a wellknown correspondence between convex polytopes in the dual space $V^{*}$ and piecewise linear convex functions on $V$. Namely, to any convex polytope, we associate its support function. Denote by $R$ the algebra generated by the support functions of all convex polytopes, in the algebra of continuous functions on $V$. In the first section of this paper, we study the algebra $R$ when $K$ is the field of rational numbers. It turns out (see 1.5) that $R$ is the algebra of continuous, piecewise polynomial functions on $V$; in particular, $R$ contains the algebra of polynomial functions. We prove that any choice of coordinate functions on $V$ defines a regular sequence in $R$; moreover, the quotient of $R$ by the ideal generated by $V^{*}$ is isomorphic to the rational polytope algebra of McMullen (see 1.3, 1.5; our proof is based on work of Morelli, see [21]). This explains the non-trivial grading of the polytope algebra, by the obvious grading of $R$. More generally, the quotients of $R$ by powers of the ideal generated by $V^{*}$, are isomorphic to the higher versions of the polytope algebra, considered recently by McMullen, Pukhlikov and Khovanskii; see [20], [24].

In fact, we study the algebra $R$ as the direct limit of its subalgebras $R_{\Sigma}$ consisting of functions which are piecewise polynomial with respect to a fixed fan $\Sigma$. To such a fan is

1991 Mathematics Subject Classification: 14M25, 52B50.

The paper is in final form and no version of it will be published elsewhere. 
associated a toric variety $X_{\Sigma}$. In the second and third sections of this paper, we identify $R_{\Sigma}$ with the equivariant cohomology algebra of $X_{\Sigma}$ (see 2.2); moreover, the quotient of $R_{\Sigma}$ by the ideal generated by $V^{*}$ identifies with the rational Chow ring of $X_{\Sigma}$ (see 3.1; here $X_{\Sigma}$ is assumed to be smooth, but not necessarily complete). In this way, we recover the result of Fulton and Sturmfels mentioned above, as well as the Jurkiewicz-Danilov presentation of the Chow ring of a smooth, complete toric variety.

Consider a regular subdivision $\Sigma^{\prime}$ of a regular fan $\Sigma$, and denote by $\pi: X_{\Sigma^{\prime}} \rightarrow X_{\Sigma}$ the associated toric morphism. Then the maps $\pi_{*}$ and $\pi^{*}$ between Chow rings lift canonically to equivariant cohomology (see 2.3 and 3.2). Of course, the pull-back $\pi^{*}: R_{\Sigma} \rightarrow R_{\Sigma^{\prime}}$ turns out to be the inclusion of $R_{\Sigma}$ into $R_{\Sigma^{\prime}}$. But the push-forward $\pi_{*}: R_{\Sigma^{\prime}} \rightarrow R_{\Sigma}$ is more subtle: it provides a canonical way of "smoothing" piecewise polynomial functions. We compute $\pi_{*}$ in 2.3, and we find relations between push-forward, Fourier transform and mixed volume in 2.4. Using the push-forward, we generalize Hadwiger's characterization of the volume of convex polytopes (see [19] Section 7) to a characterization of the integral of polynomial functions on convex polytopes. On the other hand, the push-forward determines the "intersection form" on the Chow rings of smooth, complete torus embeddings. In this way, we recover the above-mentioned theorem of Bernstein and Kouchnirenko (see 3.3).

This theorem is generalized from the torus $\left(\mathbf{C}^{*}\right)^{d}$ to any spherical homogeneous space, in the fourth section of this paper. Namely, to any effective divisor in such a space, we associate a "Newton polytope" (a convex polytope with rational vertices). Moreover, we compute the number of common points to effective divisors in general position, in terms of their Newton polytopes. For this we use a "mixed integral" which generalizes the mixed volume occurring in Kouchnirenko's theorem. Our result is a refinement of [8] where an integral formula was obtained for the degree of ample divisors on projective spherical varieties. Another generalization of Bernstein-Kouchnirenko's theorem has been obtained by B. Y. Kazarnovskii (see [16]); it holds in any connected reductive group $\Gamma$. Observe that Kazarnovskii's result can be deduced from ours, because $\Gamma$ is a spherical homogeneous space under the action of $\Gamma \times \Gamma$ by left and right multiplication. Our Bézout theorem can also be applied to the space of smooth quadrics of rank $d$ in $\mathbf{P}^{r}$; namely, this space is homogeneous and spherical under $\operatorname{PGL}(r+1)$. In the case of plane conics $(d=r=2)$, classical results of Halphen, updated and generalized by Casas and Xambó (see [9]), can be explained in our framework. It turns out that our notion of a Newton polytope is closely related to Halphen's first formula (see [9] and [23]). Moreover, our Bézout theorem specializes to Halphen's second formula. These connections will be developed elsewhere.

A technical point of the paper is the distinction between embeddings of a torus $T$ (i.e. normal varieties where $T$ acts faithfully with a dense orbit) and what we call toric varieties (the action of $T$ need no longer be faithful, but its kernel is connected). Toric varieties are classified by generalized fans; the cones in a generalized fan need not be acute, but all cones have the same linear part. These technicalities are required at several points, in particular in the study of the push-forward.

Note added in proof (December 1995). For extensions of the results of Section 1 to convex polytopes over an arbitrary subfield of $\mathbf{R}$, see my preprint The structure of the polytope algebra. For a study of equivariant $K$-theory and equivariant cohomology of simplicial toric varieties, with applications to an Euler-MacLaurin summation formula for convex lattice polytopes, see my joint preprint with Michèle Vergne An equivariant Riemann-Roch theorem for complete simplicial toric varieties. 


\section{Piecewise polynomial functions.}

1.1. Notation. Let $d$ be a positive integer. Let $N$ be a free abelian group of rank $d$; set $N_{\mathbf{Q}}=N \otimes_{\mathbf{Z}} \mathbf{Q}$. We denote by $M$ the dual lattice of $N$, and by $M_{\mathbf{Q}}$ the dual vector space of $N_{\mathrm{Q}}$.

A closed half-space in $N_{\mathbf{Q}}$ is defined by an inequality $m \geq 0$ where $m$ is a non-zero element of $M_{\mathbf{Q}}$. We normalize $m$ by assuming that $m \in M$ and that $m$ is not divisible in $M$.

A (polyhedral convex) cone $\sigma$ in $N_{\mathbf{Q}}$ is the intersection of finitely many closed halfspaces. If we write $\sigma=\bigcap_{i=1}^{r}\left(m_{i} \geq 0\right)$ with all $m_{i}$ normalized as before and with $r$ minimal, then the set $\left\{m_{1}, \ldots, m_{r}\right\}$ is uniquely determined. The hyperplanes $\left(m_{i}=0\right)$ are called the walls of $\sigma$. The largest linear subspace of $\sigma$ is $l(\sigma):=\bigcap_{i=1}^{r}\left(m_{i}=0\right)$. On the other hand, the smallest linear subspace which contains $\sigma$ is $L(\sigma)=\sigma-\sigma$. We call $\sigma$ simplicial if $m_{1}, \ldots, m_{r}$ are linearly independent, i.e. if $r=\operatorname{codim}(\sigma)$. When $m_{1}, \ldots, m_{r}$ can be completed to a basis of $M$, the cone $\sigma$ is called regular.

A generalized fan in $N_{\mathbf{Q}}$ is given by a linear subspace $l$ of $N_{\mathbf{Q}}$ and a finite set $\Sigma$ of cones, such that:

(i) $l(\sigma)=l$ for any $\sigma \in \Sigma$.

(ii) If $\sigma \in \Sigma$ and $\tau$ is a face of $\sigma$, then $\tau \in \Sigma$.

(iii) If $\sigma, \tau \in \Sigma$ then $\sigma \cap \tau$ is a face of $\sigma$.

When $l=0$, we recover the usual notion of a fan. For arbitrary $l$, a generalized fan consists in the preimages in $N_{\mathbf{Q}}$ of an ordinary fan in $N_{\mathbf{Q}} / l$.

The support $|\Sigma|$ of the generalized fan $\Sigma$ is the union of its cones. A mapping $f$ : $|\Sigma| \rightarrow \mathbf{Q}$ is piecewise polynomial if for any $\sigma \in \Sigma$, the map $\left.f\right|_{\sigma}: \sigma \rightarrow \mathbf{Q}$ extends to a polynomial function on the linear space $L(\sigma)$. In other words, a piecewise polynomial function $f$ on $\Sigma$ is a collection of polynomial functions $f_{\sigma}: \sigma \rightarrow \mathbf{Q}$ which are compatible in an obvious sense. In particular, such a function is continuous. Furthermore, if $f=\left(f_{\sigma}\right)$ is piecewise polynomial and $n$ is a non-negative integer, then the collection $\left(f_{\sigma, n}\right)$ of all homogeneous components of degree $n$ of the $f_{\sigma}$ 's is piecewise polynomial, too. Equivalently, any piecewise polynomial function decomposes uniquely as a sum of homogeneous piecewise polynomial functions.

We denote by $R_{\Sigma}$ the set of all piecewise polynomial functions on $\Sigma$. Then $R_{\Sigma}$ is a ring for the operations of pointwise addition and multiplication. We denote by $S^{\bullet}\left(M_{\mathbf{Q}}\right)$ the symmetric algebra of $M_{\mathbf{Q}}$, i.e. the algebra of polynomial functions on $N_{\mathbf{Q}}$; another notation for $S^{\bullet}\left(M_{\mathbf{Q}}\right)$ is $R_{N}$. The decomposition of piecewise polynomial functions as sums of their homogeneous components defines a grading on $R_{\Sigma}$, with $S^{\bullet}\left(M_{\mathbf{Q}}\right)=R_{N}$ as a graded subalgebra.

1.2. The algebra of piecewise polynomial functions. Let $\Sigma$ be a simplicial generalized fan in $N_{\mathbf{Q}}$, and let $\tau \in \Sigma$ be a minimal cone. Then we have $\operatorname{dim}(\tau)=1+\operatorname{dim}(l)$ where $l$ is the linear space associated to $\Sigma$. Therefore, the additive semigroup $(\tau \cap N) /(l \cap N)$ has a unique generator. We denote by $n_{\tau}$ a representative of this generator in $\tau \cap N$.

If $\sigma \in \Sigma$ contains $\tau$, then there exists a unique maximal face $\tau^{\prime}$ of $\sigma$ which does not contain $\tau$. Furthermore, there exists a unique linear form $\varphi_{\sigma, \tau}: L(\sigma) \rightarrow \mathbf{Q}$ such that $\varphi_{\tau, \sigma}$ is identically zero on $\tau^{\prime}$ and that $\varphi_{\tau, \sigma}\left(n_{\tau}\right)=1$. By the first condition, $\varphi_{\tau, \sigma}$ vanishes on $l$; it follows that $\varphi_{\tau, \sigma}$ does not depend on the choice of $n_{\tau}$. Moreover, $\varphi_{\tau, \sigma}$ is non-negative on $\sigma$.

The linear forms $\varphi_{\tau, \sigma}$ glue together to a piecewise linear function $\varphi_{\tau}$ on the star of $\tau$; 
observe that $\varphi_{\tau}$ is invariant under translation by $l$. Moreover, $\varphi_{\tau}$ is zero on the boundary of the star of $\tau$. So we have constructed $\varphi_{\tau} \in R_{\Sigma}$. It is easy to see that the $\varphi_{\tau}(\tau$ a minimal cone in $\Sigma$ ) form a basis of the space of piecewise linear functions on $\Sigma$ which are invariant under translation by $l$.

For any cone $\sigma \in \Sigma$, we set $\varphi_{\sigma}=\prod_{\tau} \varphi_{\tau}$ (product over all minimal cones $\tau \subset \sigma$ ). Then $\varphi_{\sigma}$ is homogeneous of degree $\operatorname{dim}(\sigma)-\operatorname{dim}(l)$, and invariant under translation by $l$. Moreover, the support of $\varphi_{\sigma}$ is the star of $\sigma$. If $\sigma$ is a maximal cone in $\Sigma$, then $\varphi_{\sigma}$ is the product of the equations of its walls, up to some constant multiplicative term.

Proposition. For any simplicial generalized fan $\Sigma$, and for any maximal cone $\sigma \in \Sigma$, restriction to $\Sigma \backslash\{\sigma\}$ defines an exact sequence

$$
0 \rightarrow \varphi_{\sigma} R_{L(\sigma)} \rightarrow R_{\Sigma} \rightarrow R_{\Sigma \backslash\{\sigma\}} \rightarrow 0 .
$$

Pr o of. First of all, observe that the support of $\varphi_{\sigma}$ is $\sigma$. Therefore, we have in $R_{\Sigma}$ that $\varphi_{\sigma} R_{\Sigma}=\varphi_{\sigma} R_{L(\sigma)}$ and this subset is mapped to zero by restriction to $R_{\Sigma \backslash\{\sigma\}}$. Conversely, if $f \in R_{\Sigma}$ restricts to zero on $R_{\Sigma \backslash\{\sigma\}}$, then $f_{\sigma}$ is divisible in $R_{L(\sigma)}$ by the equation of each wall of $\sigma$. Hence $f_{\sigma} \in \varphi_{\sigma} R_{L(\sigma)}$ and our sequence is left exact.

To check the surjectivity of restriction, it is enough to show that any piecewise polynomial function $\left(f_{\tau}\right)_{\tau \subset \sigma}$ on the boundary of $\sigma$, extends to a polynomial function on $\sigma$. Choose coordinates $x_{1}, \ldots, x_{d}$ on $N_{\mathrm{Q}}$ such that $\sigma=\bigcap_{i=1}^{r}\left(x_{i} \geq 0\right)$. By assumption, for any proper subset $I$ of $\{1, \ldots, r\}$, we have a polynomial function $f_{I}\left(x_{i}\right)_{i \in I}$ on the face $\tau_{I}=\sigma \cap \bigcap_{i \notin I}\left(x_{i}=0\right)$. Now set

$$
f\left(x_{1}, \ldots, x_{d}\right)=\sum_{I}(-1)^{r-1-\operatorname{card}(I)} f_{I}\left(x_{i}\right)_{i \in I} .
$$

Then $f$ is the desired extension.

By induction over the number of maximal cones in $\Sigma$, we obtain the following result, which is essentially due to L. J. Billera (see [5] 3.17 and 4.4).

COROLlary. (i) For any simplicial generalized fan $\Sigma$, the $S^{\bullet}\left(M_{\mathbf{Q}}\right)$-module $R_{\Sigma}$ is generated by the $\varphi_{\sigma}, \sigma \in \Sigma$. In particular, the $S^{\bullet}\left(M_{\mathbf{Q}}\right)$-module $R_{\Sigma}$ is finite, and the graded algebra $R_{\Sigma}$ is generated by finitely many elements of degree 1.

(ii) If moreover $\Sigma^{\prime}$ is a generalized sub-fan of $\Sigma$, then the restriction $R_{\Sigma} \rightarrow R_{\Sigma^{\prime}}$ is surjective.

(iii) The Hilbert series of $R_{\Sigma}$ is

$$
\sum_{n=0}^{\infty} \operatorname{dim}\left(R_{\Sigma, n}\right) z^{n}=\sum_{\sigma \in \Sigma} z^{\operatorname{dim}(\sigma)-\operatorname{dim}(l)}(1-z)^{-\operatorname{dim}(\sigma)} .
$$

(iv) The Krull dimension of the algebra $R_{\Sigma}$ is the maximal dimension of cones in $\Sigma$.

1.3. Relation with the Reisner-Stanley algebra. Let $\Sigma$ be a simplicial generalized fan, with its associated linear space $l$. Define the Reisner-Stanley algebra $\mathcal{R}_{\Sigma}$ to be the (commutative, associative) Q-algebra with generators $x_{\tau}(\tau$ a minimal cone of $\Sigma)$ and relations $\prod_{i=1}^{n} x_{\tau_{i}}=0$ whenever $\tau_{1}, \ldots, \tau_{n}$ are distinct minimal cones, which do not generate a cone of $\Sigma$. Clearly, $\prod_{i=1}^{n} \varphi_{\tau_{i}}=0$ in this case. Therefore, there is a unique algebra homomorphism from $\mathcal{R}_{\Sigma}$ to $R_{\Sigma}$, which sends $x_{\tau}$ to $\varphi_{\tau}$. This homomorphism is bijective when $\Sigma$ is a fan; see [5] 2.3 and 3.6, and also [6] 4.2. Let us recover this result, and extend it to generalized fans. 
Choose a direct sum decomposition $N=(l \cap N) \oplus S$, hence an injection $R_{l} \rightarrow R_{N}$ and an algebra homomorphism $h_{\Sigma}: R_{l} \otimes \mathcal{R}_{\Sigma} \rightarrow R_{\Sigma}$.

Proposition. Notation being as above, the map $h_{\Sigma}$ is an isomorphism.

Proof. First observe that $\Sigma \cap S$ is a fan, and that each cone $\sigma \in \Sigma$ decomposes as $\sigma=l \oplus(\sigma \cap S)$. It follows easily that $R_{\Sigma} \simeq R_{l} \otimes R_{\Sigma \cap S}$. Therefore, we can assume that $l=0$, i.e. that $\Sigma$ is a fan.

First assume that $\Sigma$ has only one maximal cone $\sigma$. Then $R_{\Sigma} \simeq R_{L(\sigma)}$. On the other hand, the algebra $\mathcal{R}_{\Sigma}$ is freely generated by the equations of the walls of $\sigma$, so our statement holds in this case.

In the general case, we choose a maximal cone $\sigma \in \Sigma$ and we consider the diagram

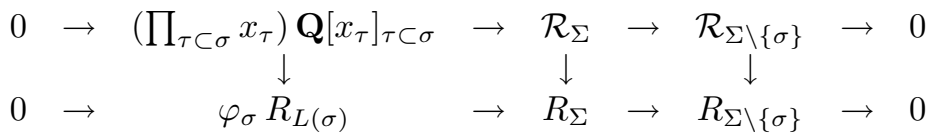

This diagram commutes, its rows are exact, and the left vertical map is an isomorphism. By induction, we may assume that the right vertical arrow $h_{\Sigma \backslash\{\sigma\}}$ is an isomorphism. Therefore, $h_{\Sigma}$ is, too.

Using [25] II.3 and II.4, we obtain easily the following result, which can also be deduced from [6] 4.5 .

COROLlary. Let $\Sigma$ be a simplicial generalized fan whose support is convex, of dimension d. Then the algebra $R_{\Sigma}$ is Cohen-Macaulay, and any basis of $M_{\mathbf{Q}}$ is a regular sequence in $R_{\Sigma}$. In particular, the $S^{\bullet}\left(M_{\mathbf{Q}}\right)$-module $R_{\Sigma}$ is free.

1.4. The completion of the algebra of piecewise polynomial functions. Let $\Sigma$ be a simplicial generalized fan. For any integer $n$, we denote by $R_{\Sigma, n}$ the component of $R_{\Sigma}$ of degree $n$, and we set

$$
R_{\Sigma, \geq n}:=\bigoplus_{m \geq n} R_{\Sigma, n} .
$$

This defines a decreasing filtration of $R_{\Sigma}$; we denote by $\hat{R}_{\Sigma}$ the associated completion.

Denote by $M^{n} R_{\Sigma}$ the ideal of $R_{\Sigma}$ generated by all products of $n$ linear forms. Clearly, we have $R_{\Sigma, \geq n} \supset M^{n} R_{\Sigma}$ for any $n \geq 0$. We will obtain an opposite inclusion, and a description of the ring $\hat{R}_{\Sigma}$ as well. First observe that $\hat{R}_{N}$ is the ring of formal power series in $d$ variables, with rational coefficients.

Proposition. (i) For any $n \geq 0$, we have $R_{\Sigma, \geq n+d} \subset M^{n} R_{\Sigma}$.

(ii) The filtration $\left(R_{\Sigma, \geq n}\right)$ is equivalent to the filtration by powers of the ideal $M R_{\Sigma}$.

(iii) The ring $\hat{R}_{\Sigma}$ is isomorphic to the subring of $\bigoplus_{\sigma \in \Sigma} \hat{R}_{L(\sigma)}$ consisting of all $\left(f_{\sigma}\right)_{\sigma \in \Sigma}$ such that $\left.f_{\sigma}\right|_{\tau}=f_{\tau}$ whenever $\tau \subset \sigma$.

Proof. (i) We argue by induction over the number of cones in $\Sigma$. In the case of one cone, we have $M^{n} R_{\Sigma}=R_{\Sigma, \geq n}$. In general, let $\sigma$ be a maximal cone in $\Sigma$. By Proposition 1.2 and the induction hypothesis, we have

$$
R_{\Sigma, \geq n+d} \subset M^{n} R_{\Sigma}+\varphi_{\sigma} R_{L(\sigma), \geq n+d-\operatorname{deg}\left(\varphi_{\sigma}\right)} .
$$

Moreover, we have

$$
\varphi_{\sigma} R_{L(\sigma), \geq n+d-\operatorname{deg}\left(\varphi_{\sigma}\right)} \subset \varphi_{\sigma} R_{L(\sigma), \geq n}=\varphi_{\sigma} M^{n} R_{N} \subset M^{n} R_{\Sigma} .
$$

(ii) is obvious now. 
(iii) We set

$$
S_{\Sigma}:=\left\{\left(f_{\sigma}\right) \in \bigoplus_{\sigma \in \Sigma} \hat{R}_{L(\sigma)}\left|f_{\sigma}\right|_{\tau}=f_{\tau} \forall \tau \subset \sigma\right\}
$$

Clearly, the ring $\hat{R}_{\Sigma}$ embeds into $S_{\Sigma}$, and this embedding commutes with restriction to any generalized sub-fan. Moreover, the analogue of Proposition 1.2 holds for $S_{\Sigma}$, with the same proof. On the other hand, the exact sequence in 1.2 involves only finitely generated $S^{\bullet}\left(M_{\mathrm{Q}}\right)$-modules. By completing this sequence with respect to the $M R_{\Sigma}$-adic topology, we obtain an exact sequence

$$
0 \rightarrow \varphi_{\sigma} \hat{R}_{L(\sigma)} \rightarrow \hat{R}_{\Sigma} \rightarrow \hat{R}_{\Sigma \backslash\{\sigma\}} \rightarrow 0 .
$$

Now the equality of $\hat{R}_{\Sigma}$ and $S_{\Sigma}$ follows by the standard argument.

1.5. Relation with the polytope algebra. Denote by $\mathcal{P}$ the set of all convex polytopes in $M_{\mathbf{Q}}$. Let $\tilde{\Pi}$ be the quotient of the free $\mathbf{Q}$-vector space on $\mathcal{P}$ by the relations $[P]+[Q]-$ $[P \cup Q]-[P \cap Q]$ whenever $P, Q$ and $P \cup Q$ are in $\mathcal{P}$. The Minkowski sum of polytopes endowes $\tilde{\Pi}$ with a ring structure. The additive group $M_{\mathbf{Q}}$ acts on $\mathcal{P}$ by translations, and this gives rise to an action of $M_{\mathbf{Q}}$ on $\tilde{\Pi}$ by automorphisms.

We denote by $A=\mathbf{Q}\left[M_{\mathbf{Q}}\right]$ the algebra of the group $M_{\mathbf{Q}}$ over $\mathbf{Q}$, and by $I$ the augmentation ideal of $A$. The canonical basis of $A$ is denoted by $\left(e^{m}\right)\left(m \in M_{\mathbf{Q}}\right)$; then the $\mathbf{Q}$-vector space $I$ is generated by the $e^{m}-1\left(m \in M_{\mathbf{Q}}\right)$. The ring $\tilde{\Pi}$ is an algebra over $A$; the quotient $\tilde{\Pi} / I \tilde{\Pi}:=\Pi$ is a version of the polytope algebra (see [19], [21], [14]).

Denote by $R$ the direct limit of the algebras $R_{\Sigma}$ for all complete, regular fans $\Sigma$. Then $R$ is the algebra of piecewise polynomial functions on $N_{\mathbf{Q}}$. Similarly, we define $\hat{R}$ to be the direct limit of the algebras $\hat{R}_{\Sigma}$. Every polytope $P \in \mathcal{P}$ defines an element of $R$, as follows. Introduce the support function

$$
\begin{array}{cccc}
H_{P}: \quad N_{\mathbf{Q}} & \rightarrow & \mathbf{Q} \\
n & \rightarrow \min _{m \in P}\langle m, n\rangle .
\end{array}
$$

Then $H_{P}$ is an element of degree 1 in $R$. Observe that the vector space $R$ is generated by the non-negative powers of support functions of all convex polytopes. Namely, the algebra $R$ is generated by its elements of degree 1 , i.e. by the piecewise linear functions (this follows from Corollary 1.2 and from the fact that any $\varphi_{\sigma}$ is a product of elements of degree 1). Moreover, any piecewise linear function is the difference of two convex piecewise linear functions.

To any $P \in \mathcal{P}$ we associate the formal exponential of its support function

$$
\exp \left(H_{P}\right)=\sum_{n=0}^{\infty} H_{P}^{n} / n ! .
$$

This exponential can be seen as an element of $\hat{R}$; denote it by $\gamma(P)$. Observe that $\gamma(P+Q)=\gamma(P) \gamma(Q)$ (because $\left.H_{P+Q}=H_{P}+H_{Q}\right)$. Therefore, $\gamma$ induces an algebra homomorphism $\gamma: \tilde{\Pi} \rightarrow \hat{R}$.

THEOREM. Notation being as above, the homomorphism $\gamma: \tilde{\Pi} \rightarrow \hat{R}$ is injective and it sends $I^{n} \tilde{\Pi}$ to $M^{n} \hat{R}$ for any $n \geq 1$. Moreover, the induced morphism

$$
\gamma_{n}: \tilde{\Pi} / I^{n} \tilde{\Pi} \rightarrow \hat{R} / M^{n} \hat{R}=R / M^{n} R
$$

is an isomorphism. 
In particular, the polytope algebra is isomorphic to $R / M R$. By work of McMullen, the action of the group of dilations on $\mathcal{P}$ leads to a grading of $\Pi$ by integers between 0 and $d$. More generally, the quotient $\tilde{\Pi} / I^{n+1} \tilde{\Pi}$ is graded by integers between 0 and $n+d$. This can be verified by using the theorem above, together with Proposition 1.4 (i). The component of highest degree in $\tilde{\Pi} / I^{n+1} \tilde{\Pi}$ will be described in 2.5 below.

P r o of. First consider the restriction of $\gamma$ to $A$, i.e.

$$
\begin{array}{cccc}
\delta: \quad A & \rightarrow & \hat{R}_{N} \\
e^{m} & \rightarrow & \exp (m) .
\end{array}
$$

Clearly, $\delta$ is injective. Moreover, $\delta$ sends $I^{n}$ to $M^{n} \hat{R}_{N}$ (because the power series $\left(e^{m}-1\right) / m$ is invertible for any $m \neq 0)$. Introduce the map

$$
\begin{aligned}
\varphi: M_{\mathbf{Q}} & \rightarrow I / I^{2} \\
m & \rightarrow 1-e^{m} \quad\left(\bmod I^{2}\right) .
\end{aligned}
$$

It is easy to check that $\varphi$ is an isomorphism of $\mathbf{Q}$-vector spaces, and that $\varphi$ induces an isomorphism

It follows that the map

$$
\varphi: S^{\bullet}\left(M_{\mathbf{Q}}\right) \rightarrow \bigoplus_{n=0}^{\infty} I^{n} / I^{n+1}
$$

$$
\operatorname{gr} \delta: \bigoplus_{n=0}^{\infty} I^{n} / I^{n+1} \rightarrow \bigoplus_{n=0}^{\infty} M^{n} \hat{R}_{N} / M^{n+1} \hat{R}_{N}=S^{\bullet}\left(M_{\mathbf{Q}}\right)
$$

is an isomorphism; namely, $\varphi$ is the inverse map of $\operatorname{gr} \delta$. So $\delta$ induces isomorphisms $A / I^{n} \rightarrow \hat{R}_{N} / M^{n} \hat{R}_{N}=R_{N} / M^{n} R_{N}$ for any $n \geq 1$. Hence, we have $I^{n}=A \cap M^{n} \hat{R}_{N}$.

Now consider a regular fan $\Sigma$. For any $\sigma \in \Sigma$, set

$$
A_{\sigma}=\mathbf{Q}\left[M_{\mathbf{Q}} / \sigma^{\perp}\right]
$$

(the algebra of the group $M_{\mathbf{Q}} / \sigma^{\perp}=L(\sigma)^{*}$ over the field of rational numbers). There is a canonical restriction map $A_{\sigma} \rightarrow A_{\tau}$ whenever $\tau \subset \sigma$. We set

$$
A_{\Sigma}=\left\{\left(f_{\sigma}\right)_{\sigma \in \Sigma} \mid f_{\sigma} \in A_{\sigma} \text { and } f_{\sigma} \mid \tau=f_{\tau} \forall \tau \subset \sigma\right\} .
$$

Then $A_{\Sigma}$ is an algebra over $A$. By the first part of the proof, each $A_{\sigma}$ is contained in $\hat{R}_{\sigma}$, and moreover $\hat{R}_{\sigma}$ is the $I$-adic completion of $A_{\sigma}$. So it follows from 1.4 that $A_{\Sigma}$ is contained in $\hat{R}_{\Sigma}$. Moreover, we have $I^{n} A_{\Sigma} \subset M^{n} \hat{R}_{\Sigma}$ by the first part of the proof.

We claim that $\hat{R}_{\Sigma}$ is the $I$-adic completion of the $A$-module $A_{\Sigma}$. The proof of this claim requires some care, because the $\operatorname{ring} A$ is not Noetherian.

For any maximal cone $\sigma$ in $\Sigma$, we have an exact sequence of $A$-modules (as in 1.2 )

$$
0 \rightarrow \Phi_{\sigma} A_{\sigma} \rightarrow A_{\Sigma} \rightarrow A_{\Sigma \backslash\{\sigma\}} \rightarrow 0
$$

where $\Phi_{\sigma}$ denotes the product of the $1-e^{m}$ over all walls $m$ of $\sigma$. Observe that $\Phi_{\sigma} / \varphi_{\sigma}$ is a unit in $\hat{R}_{N}$. Moreover, we have (by 1.3 and 1.4)

$$
\left(I^{n} A_{\Sigma}\right) \cap\left(\Phi_{\sigma} A_{\sigma}\right) \subset\left(M^{n} \hat{R}_{\Sigma}\right) \cap\left(\varphi_{\sigma} \hat{R}_{\sigma}\right) \subset M^{n-d} \varphi_{\sigma} \hat{R}_{\sigma}
$$

and therefore

$$
\left(I^{n} A_{\Sigma}\right) \cap\left(\Phi_{\sigma} A_{\sigma}\right) \subset \Phi_{\sigma}\left(A_{\sigma} \cap M^{n-d} \hat{R}_{\sigma}\right)=I^{n-d} \Phi_{\sigma} A_{\sigma} .
$$

So the $I$-adic topology on $A_{\Sigma}$ induces the $I$-adic topology on $\Phi_{\sigma} A_{\sigma}$. Therefore, the sequence of $I$-adic completions

$$
0 \rightarrow \Phi_{\sigma} \hat{A}_{\sigma} \rightarrow \hat{A}_{\Sigma} \rightarrow \hat{A}_{\Sigma \backslash\{\sigma\}} \rightarrow 0
$$

is exact. Now our claim is proved by the standard argument. 
Using [11] 7.2.12, it follows that the maps $A_{\Sigma} / I^{n} A_{\Sigma} \rightarrow R_{\Sigma} / M^{n} R_{\Sigma}$ are isomorphisms. To conclude the proof, we use the following result of Morelli (see [21] Section 5 and also [22] 4.5). Consider the map

$$
\begin{aligned}
& \mathcal{P} \rightarrow \lim A_{\Sigma} \\
& P \rightarrow e^{H_{P}}
\end{aligned}
$$

where $\lim A_{\Sigma}$ denotes the direct limit of the algebras $A_{\Sigma}$ associated to all complete, regular fans $\Sigma$. Then the induced map $\tilde{\Pi} \rightarrow \lim A_{\Sigma}$ is an isomorphism.

\section{Toric varieties and their equivariant cohomology.}

2.1. Generalized fans and toric varieties. Denote by $T=\operatorname{Hom}\left(M, \mathbf{C}^{*}\right)$ the algebraic torus with character group $M$; then $N$ is the group of one-parameter subgroups of $T$. A normal algebraic variety $X$ where $T$ acts faithfully with a dense orbit, is called a torus embedding. If we do not require that $T$ acts faithfully, but only that the kernel of the action is connected, then we call $X$ a toric variety.

Torus embeddings (resp. toric varieties) are classified by fans (resp. generalized fans) as follows. Choose a point $x$ in the open $T$-orbit of the toric variety $X$. Let $Y$ be a $T$-orbit in $X$. Consider the set of all $\lambda \in N$ such that $\lim _{t \rightarrow 0} \lambda(t) x$ exists and belongs to $Y$. This set is a semi-group, which generates a cone $\sigma_{Y}$ in $N_{\mathbf{Q}}$ (the semi-group is the intersection of $N$ with the relative interior of $\sigma_{Y}$ ). It is easy to see that the $\sigma_{Y}$ ( $Y$ a $T$-orbit in $X$ ) patch together into a generalized fan $\Sigma_{X}$. The linear part $l$ of each cone of $\Sigma_{X}$ is generated by the one-parameter subgroups which act trivially on $X$.

Conversely, given a cone $\sigma \in \Sigma$, we denote by $\check{\sigma} \subset M_{\mathbf{Q}}$ its dual cone, and by $X_{\sigma}$ the affine algebraic variety whose coordinate ring is the algebra of the semigroup $\check{\sigma} \cap M$. Then $T$ acts on $X_{\sigma}$ with a connected kernel $T_{\sigma}:=\bigcap_{m \in \check{\sigma} \cap M} \operatorname{ker}(m)$. Moreover, the $X_{\sigma}(\sigma \in \Sigma)$ can be glued together to a toric variety $X_{\Sigma}$, and the assignments $X \rightarrow \Sigma_{X}, \Sigma \rightarrow X_{\Sigma}$ are mutually inverse. A toric variety is smooth (resp. has only quotient singularities) if and only if its fan consists in regular (resp. simplicial) cones.

There is a bijection between $\Sigma$ and the set of $T$-orbits in $X_{\Sigma}$. Namely, for any $\sigma \in \Sigma$, the affine toric variety $X_{\sigma}$ contains a unique closed orbit $\mathcal{O}_{\sigma}$. The linear space $L(\sigma)$ is generated by the one-parameter subgroups which act trivially on $\mathcal{O}_{\sigma}$. It follows that $\operatorname{dim}\left(\mathcal{O}_{\sigma}\right)=d-\operatorname{dim} L(\sigma)=\operatorname{codim}(\sigma)$. We denote by $F(\sigma)$ the closure of $\mathcal{O}_{\sigma}$ in $X_{\Sigma}$. The map $\sigma \rightarrow F(\sigma)$ is an order-reversing bijection from $\Sigma$ to the set of all irreducible, closed and $T$-stable subvarieties of $X_{\Sigma}$.

Proposition. (i) Given two toric varieties $X, X^{\prime}$ with generalized fans $\Sigma, \Sigma^{\prime}$, there is a bijection $\pi \rightarrow \mu_{\pi}$ between equivariant morphisms $\pi: X^{\prime} \rightarrow X$, and order-preserving maps $\mu: \Sigma^{\prime} \rightarrow \Sigma$ which satisfy

$$
\sigma^{\prime} \subset \mu\left(\sigma^{\prime}\right)+L\left(\mu\left(l^{\prime}\right)\right) \forall \sigma^{\prime} \in \Sigma^{\prime}
$$

(ii) $\pi$ is dominant (resp. a closed immersion) if and only if $\mu_{\pi}\left(l^{\prime}\right)=l$ (resp. $\sigma^{\prime}=$ $\mu_{\pi}\left(\sigma^{\prime}\right)+L\left(\mu_{\pi}\left(l^{\prime}\right)\right)$ for all $\left.\sigma^{\prime} \in \Sigma^{\prime}\right)$.

(iii) $\pi$ is proper if and only if $\left|\Sigma^{\prime}\right|=|\Sigma|+l^{\prime}$.

Proof. Let $\pi: X^{\prime} \rightarrow X$ be an equivariant morphism. For any $\sigma^{\prime} \in \Sigma^{\prime}$, we have $\pi\left(\mathcal{O}_{\sigma^{\prime}}\right)=\mathcal{O}_{\sigma}$ for a unique $\sigma \in \Sigma$. We set $\mu\left(\sigma^{\prime}\right)=\sigma$ and we denote $\mu\left(l^{\prime}\right)$ by $\sigma_{0}$. Then $\pi$ factorizes as $X^{\prime} \rightarrow F\left(\sigma_{0}\right) \rightarrow X$ where the second map is the inclusion. Observe that the generalized fan of $F\left(\sigma_{0}\right)$ consists in the cones $\sigma+L\left(\sigma_{0}\right)$ for all cones in $\Sigma$ which contain $\sigma_{0}$. Moreover, $\pi$ sends the open affine subset $X_{\sigma^{\prime}}^{\prime}$ to $F\left(\sigma_{0}\right)_{\sigma}$. It follows that $\sigma^{\prime} \subset \sigma+L\left(\sigma_{0}\right)$. 
Hence $\mu$ satisfies condition $(*)$. Moreover, if $\tau^{\prime} \in \Sigma^{\prime}$ and $\tau^{\prime} \subset \sigma^{\prime}$, then $\overline{\mathcal{O}_{\tau^{\prime}}}$ contains $\mathcal{O}_{\sigma^{\prime}}$ and therefore

$$
\left.\overline{\mathcal{O}_{\mu\left(\tau^{\prime}\right)}} \supset \overline{\pi\left(\mathcal{O}_{\tau^{\prime}}\right.}\right) \supset \pi\left(\overline{\mathcal{O}_{\tau^{\prime}}}\right) \supset \mathcal{O}_{\sigma}
$$

whence $\mu\left(\tau^{\prime}\right) \subset \mu\left(\sigma^{\prime}\right)$. Conversely, the construction of a morphism from a map $\mu$ which satisfies $(*)$ is routine, as well as assertions (ii) and (iii).

2.2. Equivariant cohomology of toric varieties. Given an algebraic torus $T$, we can choose a $T$-principal bundle $E T \rightarrow B T$ such that $E T$ is contractible. If $T \simeq\left(\mathbf{C}^{*}\right)^{d}$ we can take $E T=\left(\mathbf{C}^{\infty} \backslash\{0\}\right)^{d}$ with the diagonal action of $T$, where $\mathbf{C}^{*}$ acts on $\mathbf{C}^{\infty} \backslash\{0\}$ by scalar multiplication. Then $B T=\left(\mathbf{P}^{\infty}\right)^{d}$. For any $T$-space $Z$, we construct the fiber product $Z \times_{T} E T$ over $(E T) / T=B T$. The cohomology algebra of $Z \times_{T} E T$ with rational coefficients, is called the (rational) equivariant cohomology of $Z$, and denoted by $H_{T}^{*}(Z)$. Observe that $H_{T}^{*}$ (point) $=H^{*}(B T)$ is a graded polynomial algebra on $d$ generators of degree 2. Moreover, $H_{T}^{*}(Z)$ is a graded algebra over $H^{*}(B T)$. In fact, there is a canonical isomorphism $S^{\bullet}\left(M_{\mathbf{Q}}\right) \rightarrow H^{*}(B T)$ which doubles the degree. Namely, to any $m \in M$, we associate the first Chern class of the line bundle $\mathbf{C} m \times_{T} E T$ over $B T$, where $\mathbf{C} m$ is the one-dimensional $T$-module with weight $m$. For more on equivariant cohomology, we refer to $[1]$.

Consider now a smooth toric variety $X$, its generalized fan $\Sigma$, and a cone $\sigma \in \Sigma$. Denote by $i_{\sigma}: X_{\sigma} \rightarrow X$ the inclusion of the open, affine $T$-stable subset associated to $\sigma$. The Euler class of $F(\sigma)$ (the orbit closure associated to $\sigma$ ) is an element of $H_{T}^{*}(X)$ and its degree is $\operatorname{codim} F(\sigma)=\operatorname{dim}(\sigma)-\operatorname{dim}(l)$.

Proposition. (i) The algebra $H_{T}^{*}\left(X_{\sigma}\right)$ is isomorphic to $R_{L(\sigma)}$.

(ii) The morphism

$$
\bigoplus_{\sigma \in \Sigma} i_{\sigma}^{*}: H_{T}^{*}(X) \rightarrow \bigoplus_{\sigma \in \Sigma} H_{T}^{*}\left(X_{\sigma}\right)
$$

is injective, and its image is isomorphic to $R_{\Sigma}$.

(iii) This isomorphism maps the Euler class of $F(\sigma)$ to $\varphi_{\sigma}$, for any $\sigma \in \Sigma$.

In particular, the $S^{\bullet}\left(M_{\mathbf{Q}}\right)$-algebras $H_{T}^{*}(X)$ and $R_{\Sigma}$ are isomorphic.

Pr o of. (i) Recall that $X_{\sigma}$ is the total space of an equivariant vector bundle over $\mathcal{O}_{\sigma}$ (the unique closed $T$-orbit in $X_{\sigma}$ ). Therefore, restriction to $\mathcal{O}_{\sigma}$ induces an isomorphism $H_{T}^{*}\left(X_{\sigma}\right) \rightarrow H_{T}^{*}\left(\mathcal{O}_{\sigma}\right)$. But $\mathcal{O}_{\sigma} \simeq T / T_{\sigma}$ where $T_{\sigma}$ is the subtorus of $T$ whose lattice of oneparameter subgroups is $L(\sigma) \cap N$. So we have $H_{T}^{*}\left(\mathcal{O}_{\sigma}\right)=H_{T}^{*}\left(T / T_{\sigma}\right)=H^{*}\left(B T_{\sigma}\right)=R_{L(\sigma)}$. (ii) Let $\tau$ be a face of $\sigma$; denote by $i_{\sigma \tau}: X_{\tau} \rightarrow X_{\sigma}$ the inclusion. Then we have a commutative diagram

$$
\begin{array}{ccc}
H_{T}^{*}\left(X_{\sigma}\right) & \rightarrow & H_{T}^{*}\left(X_{\tau}\right) \\
\downarrow & & \downarrow \\
R_{L(\sigma)} & \rightarrow & R_{L(\tau)}
\end{array}
$$

where the bottom horizontal map is restriction to $L(\tau)$. Therefore, the image of $i_{\sigma}^{*}$ is contained in $R_{\Sigma}$, and there is a canonical homomorphism $h_{\Sigma}: H_{T}^{*}(X) \rightarrow R_{\Sigma}$. We prove that $h_{\Sigma}$ is an isomorphism by induction over the number of cones in $\Sigma$. The case of one cone is covered by (i). In the general case, we choose a maximal cone $\sigma \in \Sigma$, and we consider the following diagram:

$$
\begin{aligned}
& 0 \quad \rightarrow \quad H_{T}^{*}\left(X_{\Sigma}, X_{\Sigma \backslash\{\sigma\}}\right) \rightarrow H_{T}^{*}\left(X_{\Sigma}\right) \rightarrow H_{T}^{*}\left(X_{\Sigma \backslash\{\sigma\}}\right) \rightarrow 0 \\
& 0 \rightarrow \varphi_{\sigma} R_{L(\sigma)} \quad \rightarrow \quad \begin{array}{cllll}
\downarrow & & & \downarrow & \\
R_{\Sigma} & \rightarrow & R_{\Sigma \backslash\{\sigma\}} & \rightarrow & 0
\end{array}
\end{aligned}
$$


By a direct generalization of [4] Lemma 3, the top line is exact, as well as the left vertical arrow. But the bottom line is exact by Proposition 1.2; the assertion follows.

(iii) The variety $F(\sigma)$ is the transversal intersection of the $F(\tau)$ for all minimal cones $\tau \subset \sigma$. On the other hand, recall that $\varphi_{\sigma}=\prod_{\tau} \varphi_{\tau}$. Therefore, we may assume that $\sigma$ is minimal; then $F(\sigma)$ is an irreducible divisor in $X$. By restricting to $X_{C}$ for some cone $C \in \Sigma$, we may assume furthermore that $X$ is affine; then $\Sigma$ consists in the faces of $C$. Let $(m=0)$ be the equation of the unique maximal face of $C$ which does not contain $\sigma$. Then the Euler class of $F(\sigma)$ is the equivariant Chern class of the line bundle on $C$ associated to $m$. So this class identifies to $m=\left.\varphi_{\sigma}\right|_{C}$.

2.3. Pull-back and push-forward. Consider two smooth toric varieties $X, X^{\prime}$ with generalized fans $\Sigma, \Sigma^{\prime}$, and an equivariant morphism $\pi: X^{\prime} \rightarrow X$. Then $\pi$ induces a morphism of graded $S^{\bullet}\left(M_{\mathbf{Q}}\right)$-algebras $\pi^{*}: H_{T}^{*}(X) \rightarrow H_{T}^{*}\left(X^{\prime}\right)$, i.e. $\pi^{*}: R_{\Sigma} \rightarrow R_{\Sigma^{\prime}}$. If moreover $\pi$ is proper, then it induces a morphism of $H_{T}^{*}(X)$-modules $\pi_{*}: H_{T}^{*}\left(X^{\prime}\right) \rightarrow$ $H_{T}^{*}(X)$ where $H_{T}^{*}(X)$ acts on $H_{T}^{*}\left(X^{\prime}\right)$ via $\pi^{*}$. So we have a morphism of $R_{\Sigma^{-m o d u l e s}}$ $\pi_{*}: R_{\Sigma^{\prime}} \rightarrow R_{\Sigma}$.

We will describe $\pi^{*}$ and $\pi_{*}$ in combinatorial terms, using the notation and results of 2.1. Let $\mu: \Sigma^{\prime} \rightarrow \Sigma$ be the map associated to $\pi$. Observe that condition $(*)$ implies that $L\left(\sigma^{\prime}\right) \subset L\left(\mu\left(\sigma^{\prime}\right)\right)$ for all $\sigma^{\prime} \subset \Sigma^{\prime}$. Therefore, any polynomial function on $\mu\left(\sigma^{\prime}\right)$ defines a polynomial function on $\sigma^{\prime}$.

TheOREM. (i) For any $f=\left(f_{\sigma}\right) \in R_{\Sigma}$, we have $\left(\pi^{*} f\right)_{\sigma^{\prime}}=f_{\mu\left(\sigma^{\prime}\right)}$.

(ii) If $\pi$ is proper, then for any $\sigma^{\prime} \in \Sigma^{\prime}$, we have

$$
\pi_{*} \varphi_{\sigma^{\prime}}= \begin{cases}\varphi_{\sigma} & \text { when } \mu\left(\sigma^{\prime}\right)=\sigma \text { and } \operatorname{dim}\left(\sigma^{\prime}\right)-\operatorname{dim}\left(l^{\prime}\right)=\operatorname{dim}(\sigma)-\operatorname{dim}(l) \\ 0 & \text { otherwise. }\end{cases}
$$

(iii) If $\pi$ is proper and all maximal cones of $\Sigma$ and $\Sigma^{\prime}$ are d-dimensional, then we have

$$
\left(\pi_{*} f\right)_{\sigma}=\varphi_{\sigma} \sum_{\mu\left(\sigma^{\prime}\right)=\sigma} \varphi_{\sigma^{\prime}}^{-1} f_{\sigma^{\prime}}
$$

for any $f=\left(f_{\sigma^{\prime}}\right) \in R_{\Sigma^{\prime}}$, and any maximal cone $\sigma \in \Sigma$.

Proof. (i) Choose $\sigma^{\prime} \in \Sigma^{\prime}$ and set $\mu\left(\sigma^{\prime}\right):=\sigma$. Then $\pi$ restricts to $p: X_{\sigma^{\prime}}^{\prime} \rightarrow X_{\sigma}$. Denote by $i: X_{\sigma} \rightarrow X$ and $i^{\prime}: X_{\sigma^{\prime}}^{\prime} \rightarrow X^{\prime}$ the inclusions. Then $\pi \circ i^{\prime}=i \circ p$. So we have

$$
\left(\pi^{*} f\right)_{\sigma^{\prime}}=i^{\prime *} \pi^{*} f=p^{*} i^{*} f=p^{*} f_{\sigma} .
$$

Therefore, we may assume that $X$ and $X^{\prime}$ are affine. Then $T$ has a unique closed orbit $\mathcal{O}_{\sigma}$ (resp. $\mathcal{O}_{\sigma^{\prime}}$ ) in $X$ (resp. $X^{\prime}$ ). Denote by $j: \mathcal{O}_{\sigma} \rightarrow X$ and $j^{\prime}: \mathcal{O}_{\sigma^{\prime}} \rightarrow X^{\prime}$ the inclusions, and by $q: \mathcal{O}_{\sigma^{\prime}} \rightarrow \mathcal{O}_{\sigma}$ the restriction of $p$. Then $j^{*}$ and $j^{\prime *}$ are isomorphisms (because $X$ is the total space of an equivariant vector bundle over $\mathcal{O}_{\sigma}$, and the same holds for $X^{\prime}$ ). Moreover, as observed in the proof of Proposition 2.2, the map $q^{*}: H_{T}^{*}\left(\mathcal{O}_{\sigma}\right) \rightarrow H_{T}^{*}\left(\mathcal{O}_{\sigma}^{\prime}\right)$ identifies with the inclusion of $R_{L(\sigma)}$ into $R_{L\left(\sigma^{\prime}\right)}$. The assertion follows.

(ii) Denote by $i: F(\sigma) \rightarrow X$ and $i^{\prime}: F\left(\sigma^{\prime}\right) \rightarrow X^{\prime}$ the inclusions, and by $p: F\left(\sigma^{\prime}\right) \rightarrow$ $F(\sigma)$ the restriction of $\pi$. Then Proposition 2.2 (iii) gives $\varphi_{\sigma^{\prime}}=i_{*}^{\prime}(1)$. Therefore, we have $\pi_{*} \varphi_{\sigma^{\prime}}=\pi_{*} i_{*}^{\prime}(1)=i_{*} p_{*}(1)$. But $p_{*}$ is homogeneous of degree

$$
\operatorname{dim} F(\sigma)-\operatorname{dim} F\left(\sigma^{\prime}\right)=\operatorname{dim}\left(\sigma^{\prime}\right)-\operatorname{dim}\left(l^{\prime}\right)-\operatorname{dim}(\sigma)+\operatorname{dim}(l) \leq 0 .
$$

Therefore, $p_{*}(1)=0$ if $\operatorname{dim} F(\sigma) \neq \operatorname{dim} F\left(\sigma^{\prime}\right)$. On the other hand, $p$ is birational when $\operatorname{dim} F(\sigma)=\operatorname{dim} F\left(\sigma^{\prime}\right)$, because all isotropy groups of toric varieties are connected. So $p_{*}(1)=1$ in this case, and $\pi_{*} \varphi_{\sigma^{\prime}}=i_{*}(1)=\varphi_{\sigma}$. 
(iii) First consider the very special case where $\pi: F(\tau) \rightarrow X$ is the inclusion of the orbit closure associated to $\tau \in \Sigma$, and where the cone $\tau$ is maximal. Then $F(\tau)$ is a fixed point, and $H_{T}^{*}(F(\tau))=R_{N}$. The map $\pi_{*}$ is $R_{N}$-linear, and it sends 1 to the Euler class of $F(\tau)$, i.e. to $\varphi_{\tau}$. Therefore, our formula holds in this case.

Now consider the general case. The set of fixed points in $X$ identifies with the set $\Sigma(d)$ of maximal cones in $\Sigma$. Denote by $i: X^{T} \rightarrow X$ and by $i^{\prime}: X^{\prime T} \rightarrow X^{\prime}$ the inclusions of the fixed point sets. By (i), the morphism $i^{*}: H_{T}^{*}(X) \rightarrow H_{T}^{*}\left(X^{T}\right)$ identifies with

$$
\begin{aligned}
R_{\Sigma} & \rightarrow \bigoplus_{\sigma \in \Sigma(d)} R_{N} \\
f & \rightarrow\left(f_{\sigma}\right)_{\sigma \in \Sigma(d)} .
\end{aligned}
$$

Therefore, $i^{*}$ is injective; in particular, the $R_{N}$-module $R_{\Sigma}$ is torsion-free. By the first step of the proof, we have

$$
i^{*} i_{*}\left(f_{\sigma}\right)_{\sigma \in \Sigma(d)}=\left(f_{\sigma} \varphi_{\sigma}\right)_{\sigma \in \Sigma(d)} .
$$

So the image of $i_{*}$ generates the $R_{N}$-module $R_{\Sigma}$ up to inversion of all equations of walls of $\Sigma$. Hence it is enough to check our formula when $f=i_{*}^{\prime} g$ for some $g=\left(g_{\sigma^{\prime}}\right)_{\sigma^{\prime} \in \Sigma^{\prime}(d)}$. Then $f_{\sigma^{\prime}}=\varphi_{\sigma^{\prime}} g_{\sigma^{\prime}}$ for all $\sigma^{\prime} \in \Sigma^{\prime}(d)$, by the first step of the proof. In this case, we have

$$
\pi_{*} f=\pi_{*} i_{*}^{\prime} g=i_{*} p_{*} g
$$

where $p: X^{\prime T} \rightarrow X^{T}$ is the restriction of $\pi$. But for any $\sigma \in \Sigma$, we have

$$
p_{*}\left(g_{\sigma^{\prime}}\right)_{\sigma}=\sum_{\mu\left(\sigma^{\prime}\right)=\sigma} g_{\sigma^{\prime}}
$$

and therefore, by the first step of the proof:

$$
\left(\pi_{*} f\right)_{\sigma}=\varphi_{\sigma} \sum_{\mu(\sigma)^{\prime}=\sigma} g_{\sigma^{\prime}}=\varphi_{\sigma} \sum_{\mu\left(\sigma^{\prime}\right)=\sigma} \varphi_{\sigma^{\prime}}^{-1} f_{\sigma^{\prime}} .
$$

2.4. Push-forward, Fourier transform and mixed volume. Let $\Sigma$ be a complete, regular fan; then $\Sigma$ is a subdivision of $N$, and therefore we have a push-forward $\left(\pi_{\Sigma}\right)_{*}: R_{\Sigma} \rightarrow$ $S^{\bullet}\left(M_{\mathrm{Q}}\right)$. Recall that $R$ denotes the algebra of piecewise polynomial functions on $N$, i.e. the direct limit of the algebras $R_{\Sigma}$ over all complete, regular fans. By functoriality, the $\left(\pi_{\Sigma}\right)_{*}$ define a push-forward $\pi_{*}: R \rightarrow S^{\bullet}\left(M_{\mathbf{Q}}\right)$.

Recall that the vector space $R$ is generated by the functions $H_{P}^{n}$ for all $d$-dimensional polytopes $P$, and all non-negative integers $n$ (see 1.5). Therefore, to determine the map $\pi_{*}: R \rightarrow S^{\bullet}\left(M_{\mathbf{Q}}\right)$, it is enough to compute all $\pi_{*}\left(H_{P}^{n}\right)$. Notation being as in 1.5 , this amounts to extending $\pi_{*}: R \rightarrow R_{N}$ to $\pi_{*}: \hat{R} \rightarrow \hat{R}_{N}$ and computing $\pi_{*} \exp \left(H_{P}\right) \in \hat{R}_{N}$.

TheOrem. Let $\pi_{*}: \hat{R} \rightarrow \hat{R}_{N}$ be the push-forward. Then for any d-dimensional convex polytope $P$, with support function $H_{P}$, the formal power series $\pi_{*} \exp \left(H_{P}\right)$ represents an entire function. Moreover, for all $v \in N_{\mathbf{Q}}$, we have

$$
\left(\pi_{*} \exp \left(H_{P}\right)\right)(v)=(-1)^{d} \int_{P} \exp \langle m, v\rangle d m .
$$

Proof. Choose a complete, regular fan $\Sigma$ such that $H_{P}$ is linear on every cone of $\Sigma$. For any $d$-dimensional cone $\sigma$ in $\Sigma$, denote by $m_{1}(\sigma) \ldots, m_{d}(\sigma)$ the equations of its walls, normalized as in 1.1. Denote by $f_{\sigma}$ the linear form $H_{P} \mid \sigma$; then $f_{\sigma}$ is some vertex 
of $P$. By 2.3 , we have

$$
\pi_{*} \exp \left(H_{P}\right)=\sum_{\sigma \in \Sigma(d)} \exp \left(f_{\sigma}\right) \prod_{i=1}^{d} m_{i}(\sigma)^{-1} .
$$

On the other hand, it follows from Théorème 3.2 in [7] that

$$
\int_{P} \exp \langle m, v\rangle d m=(-1)^{d} \sum_{\sigma \in \Sigma(d)} \exp \left(f_{\sigma}\right)(v) \prod_{i=1}^{d}\left\langle m_{i}(\sigma), v\right\rangle
$$

for any $v \in N_{\mathbf{Q}}$ outside of the walls of all maximal cones in $\Sigma$. The result follows.

Corollary. For any convex polytopes $P_{1}, \ldots, P_{d}$ with respective support functions $f_{1}, \ldots, f_{d}$, we have

where $V$ denotes the mixed volume.

$$
\pi_{*}\left(f_{1} \cdots f_{d}\right)=(-1)^{d} d ! V\left(P_{1}, \ldots, P_{d}\right)
$$

Proof. By 2.3, the map $\pi_{*}$ is homogeneous of degree $-d$. So the previous theorem implies the identity

$$
\pi_{*}\left(H_{P}^{d}\right)=(-1)^{d} d ! \int_{P} d m=(-1)^{d} d ! V(P, \ldots, P)
$$

for any $d$-dimensional convex polytope $P$. The corollary follows by polarizing this identity.

2.5. Integrals of polynomial functions on convex polytopes. Consider the algebra $R$ of piecewise polynomial functions on $N_{\mathbf{Q}}$, and its quotient $R / M^{n+1} R$ for some integer $n \geq 0$. By Proposition 1.4 (i), the degree of each non-zero element in $R / M^{n+1} R$ is at most $n+d$. Using the isomorphism

$$
R / M^{n+1} R \simeq \tilde{\Pi} / I^{n+1} \tilde{\Pi}
$$

of 1.5 , let us construct linear functionals on the component of highest degree $\left(R / M^{n+1} R\right)_{n+d}$. Namely, let $g$ be any polynomial function on $M_{\mathbf{Q}}$ which is homogeneous of degree $n$. Then the map

$$
\begin{array}{rlcc}
\int g: \quad \mathcal{P} & \rightarrow & \mathbf{Q} \\
P & \rightarrow & \int_{P} g(m) d m
\end{array}
$$

is compatible with the defining relations of $\tilde{\Pi}$. Moreover, it is easy to check that the induced map $\int g: \tilde{\Pi} \rightarrow \mathbf{Q}$ vanishes on $I^{n+1}$, and is homogeneous of degree $-n-d$. Therefore, we have defined a map

$$
\int g:\left(\tilde{\Pi} / I^{n+1} \tilde{\Pi}\right)_{n+d} \rightarrow \mathbf{Q} .
$$

When $n=0$, this construction reduces to the volume map vol $: \Pi_{d} \rightarrow \mathbf{Q}$. By [19] Section 7, this map is an isomorphism. Let us extend this result to higher degrees.

Proposition. The bilinear form

$$
\begin{aligned}
S^{n}\left(N_{\mathbf{Q}}\right) \times\left(\tilde{\Pi} / I^{n+1} \tilde{\Pi}\right)_{n+d} & \rightarrow \mathbf{Q} \\
(g,[P]) & \rightarrow \int_{P} g
\end{aligned}
$$

is non-degenerate.

In particular, the space $\left(\tilde{\Pi} / I^{n+1} \tilde{\Pi}\right)_{n+d}$ is isomorphic to the space of homogeneous polynomials of degree $n$ on $N_{\mathbf{Q}}$. 
Proof. As in 2.4, let us consider the map $\pi_{*}: R \rightarrow S^{\bullet}\left(M_{\mathbf{Q}}\right)$. Recall that $\pi_{*}$ is a morphism of $S^{\bullet}\left(M_{\mathbf{Q}}\right)$-modules of degree $-d$. Therefore, it induces a morphism of $S^{\bullet}\left(M_{\mathbf{Q}}\right)$ modules of degree $-d$

$$
\text { gr } \pi_{*}: \bigoplus_{n=0}^{\infty} M^{n} R / M^{n+1} R \rightarrow \bigoplus_{n=0}^{\infty} M^{n} / M^{n+1}=S^{\bullet}\left(M_{\mathbf{Q}}\right) .
$$

Let us check first that the restriction gr $\pi_{*}:(R / M R)_{d} \rightarrow \mathbf{Q}$ is an isomorphism. Namely, it follows from 2.4 that this map identifies with the volume map from $\Pi_{d}$ to $\mathbf{Q}$; hence our claim follows from [19] Theorem $1 \mathrm{c}$ ). Now recall that any basis of $M_{\mathbf{Q}}$ is a regular sequence in $R$ (this is a consequence of Corollary 1.3). Therefore, the natural map

$$
S^{\bullet}\left(M_{\mathbf{Q}}\right) \otimes R / M R \rightarrow \bigoplus_{n=0}^{\infty} M^{n} R / M^{n+1} R
$$

is an isomorphism of graded $S^{\bullet}\left(M_{\mathbf{Q}}\right)$-modules. So the map gr $\pi_{*}$ restricts to an isomorphism of degree $-d$

$$
\bigoplus_{n=0}^{\infty}\left(M^{n} R / M^{n+1} R\right)_{n+d} \rightarrow S^{\bullet}\left(M_{\mathbf{Q}}\right)
$$

Let $P$ be a $d$-dimensional convex polytope, and let $v \in N$. By 2.4 , we have

$$
\int_{P} \frac{\langle m, v\rangle^{n}}{n !} d m=(-1)^{d} \frac{\pi_{*}\left(H_{P}^{n+d}\right)}{(n+d) !}(v) .
$$

Remember that the non-negative powers of the functions $H_{P}$ generate the vector space $R / M^{n+1} R=\tilde{\Pi} / I^{n+1} \tilde{\Pi}$. Hence, for any $v \in N_{\mathbf{Q}}$ and $f \in R / M^{n+1} R$, the evaluation of our bilinear form at $\left(v^{n}, f\right)$ is a non-zero multiple of $\left(\pi_{*} f\right)(v)$. But the map

$$
\pi_{*}:\left(R / M^{n+1} R\right)_{n+d}=\left(M^{n} R / M^{n+1} R\right)_{n+d} \rightarrow S^{n} M_{\mathbf{Q}}
$$

is injective (because the map gr $\pi_{*}$ is injective). Therefore, our bilinear form is nondegenerate on the left. We conclude by the equality

$$
\operatorname{dim} S^{n}\left(M_{\mathbf{Q}}\right)=\operatorname{dim}\left(R / M^{n+1} R\right)_{n+d}
$$

which follows from the first part of the proof.

Our statement can be reformulated in a more concrete way, by using the language of valuations on convex polytopes. Let $\Gamma$ be an abelian group. Then a map $v: \mathcal{P} \rightarrow \Gamma$ is a valuation if $v(P \cup Q)+v(P \cap Q)=v(P)+v(Q)$ whenever $P, Q$ and $P \cup Q$ are convex polytopes. The notion of a polynomial valuation can be defined in an inductive way. Namely, a valuation is called polynomial of degree 0 if it is translation-invariant. Moreover, the valuation $v: \mathcal{P} \rightarrow \Gamma$ is called polynomial of degree at most $n$ if the valuation $P \rightarrow v(P+m)-v(P)$ is polynomial of degree at most $n-1$ for any $m \in M$.

Observe that the polynomial valuations of degree at most $n$, with values in $\Gamma$, identify with the group homomorphisms from $\tilde{\Pi} / I^{n+1} \tilde{\Pi}$ to $\Gamma$. So we are led to the following

Corollary. For any valuation $v: \mathcal{P} \rightarrow \mathbf{Q}$ which is homogeneous of degree $n+d$ and polynomial of degree $n$, there exists a unique $g \in S^{n}\left(N_{\mathbf{Q}}\right)$ such that $v=\int g$.

\section{The Chow ring of a smooth toric variety.}

3.1. Equivariant cohomology and Chow ring. Notation being as in 2.2, consider any $T$-space $Z$. Then the map $Z \times_{T} E T \rightarrow B T$ is a fibration with fiber $Z$. So we have a restriction map $H_{T}^{*}(Z)=H^{*}\left(Z \times_{T} E T\right) \rightarrow H^{*}(Z)$ which vanishes on the ideal of $H_{T}^{*}(Z)$ generated by the augmentation ideal of $H^{*}(B T)$, i.e. on $M H_{T}^{*}(Z)$. The induced map

$$
v_{Z}: H_{T}^{*}(Z) / M H_{T}^{*}(Z) \rightarrow H^{*}(Z)
$$


is not an isomorphism in general (take for example $Z=T$ where $T$ acts by multiplication). However, if $Z$ has no odd rational cohomology, then $v_{Z}$ is an isomorphism, and the image of any basis of $M$ is a regular sequence in $H_{T}^{*}(Z)$; see [4] 1.1. This leads to the JurkiewiczDanilov presentation of the Chow ring of any smooth, complete toric variety, see [3]. Namely, such a variety $Z$ has no odd rational cohomology, see e.g. [13] 5.2. Moreover, the cycle map from the Chow ring to the cohomology ring of $Z$, is an isomorphism.

We will describe the image of $v_{Z}$ when $Z$ is a smooth, but not necessarily complete, toric variety.

THEOREM. For any smooth toric variety $X$, the map

$$
v_{X}: H_{T}^{*}(X) / M H_{T}^{*}(X) \rightarrow H^{*}(X)
$$

factors through an isomorphism

$$
H_{T}^{*}(X) / M H_{T}^{*}(X) \simeq A^{*}(X)
$$

followed by $\mathrm{cl}_{X}: A^{*}(X) \rightarrow H^{*}(X)$.

Here $A^{*}(X)$ denotes the rational Chow ring of $X$, and $\mathrm{cl}_{X}$ is the cycle map; see [12] Chapter 19.

Proof. Using Proposition 1.3, it is easy to reduce to the case where $X$ is a torus embedding, i.e. $\Sigma$ is a fan. Then, again by 1.3 , the algebra $R_{\Sigma}$ is defined by generators $x_{\tau}(\tau$ an edge of $\Sigma)$ and relations $x_{\tau_{1}} \cdots x_{\tau_{n}}=0$ if $\tau_{1}, \ldots, \tau_{n}$ do not generate a cone of $\Sigma$. For any such edge $\tau$, denote by $n_{\tau}$ the generator of the semigroup $\tau \cap N$. Choose $m \in M$; then the function $\sum_{\tau}\left\langle m, n_{\tau}\right\rangle \varphi_{\tau}$ coincides with the image of $m$ in $R_{\Sigma}$. Therefore, the algebra $R_{\Sigma} / M R_{\Sigma}$ is defined by generators and relations as before, plus the relations $\sum_{\tau}\left\langle m, n_{\tau}\right\rangle x_{\tau}$ for any $m \in M$.

On the other hand, the classes of $F(\tau)$ in $A^{*}(X)$ satisfy the same relations; see [13] 3.3. Therefore, $v_{X}$ factorizes through $w_{X}: R_{\Sigma} / M R_{\Sigma} \rightarrow A^{*}(X)$ followed by $\operatorname{cl}_{X}: A^{*}(X) \rightarrow$ $H^{*}(X)$. If $X$ is complete, then $v_{X}$ is an isomorphism, and $\operatorname{cl}_{X}$ is, too. Therefore, $w_{X}$ is an isomorphism. For the general case, because every smooth torus embedding has a smooth, equivariant completion, it is enough to prove the following assertion: Let $\Sigma$ be a regular fan, and let $\sigma$ be a maximal cone in $\Sigma$. Set $\Sigma^{\prime}=\Sigma \backslash\{\sigma\}$ and $X^{\prime}=X_{\Sigma^{\prime}}$. If $w_{X}$ is an isomorphism, then so is $w_{X^{\prime}}$. But this assertion follows from the diagram

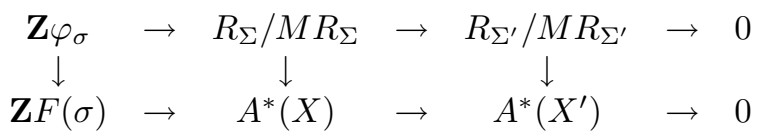

Namely, the diagram commutes, the left and middle vertical arrows are isomorphisms, and the rows are exact.

COROLlary. For any smooth toric variety $X$, with generalized fan $\Sigma$, there is an isomorphism of graded algebras $R_{\Sigma} / M R_{\Sigma} \rightarrow A^{*}(X)$ which maps the image of $\varphi_{\sigma}$ to the class of $F(\sigma)$, for any $\sigma \in \Sigma$. If moreover $\Sigma$ is a fan whose support is convex, of dimension $d$, then we have

$$
\sum_{n=0}^{d} \operatorname{dim} A^{n}(X) z^{n}=\sum_{\sigma \in \Sigma} z^{\operatorname{dim}(\sigma)}(1-z)^{\operatorname{codim}(\sigma)} .
$$

The first assertion results from the proposition above and from 2.2 ; the last assertion follows from Corollaries 1.2 (iii) and 1.3. 
3.2. Pull-back, push-forward and intersection product. As in 2.3 , consider two smooth toric varieties $X, X^{\prime}$ with generalized fans $\Sigma, \Sigma^{\prime}$, and an equivariant morphism $\pi: X^{\prime} \rightarrow$ $X$. By Corollary 3.1, we can identify $A^{*}(X)$ with $R_{\Sigma} / M R_{\Sigma}$ and $A^{*}\left(X^{\prime}\right)$ with $R_{\Sigma^{\prime}} / M R_{\Sigma^{\prime}}$.

Proposition. (i) The pull-back (for Chow rings) $\pi^{*}: A^{*}(X) \rightarrow A^{*}\left(X^{\prime}\right)$ is compatible with the pull-back defined in 2.3.

(ii) If $\pi$ is proper, then the push-forward (for Chow groups) $\pi_{*}: A_{*}\left(X^{\prime}\right) \rightarrow A_{*}(X)$ is compatible with the push-forward defined in 2.3.

Proof. (i) First consider the case where $\pi$ is an open immersion. Then $\Sigma^{\prime}$ is a generalized sub-fan of $\Sigma$, and moreover $l^{\prime}=l$. Let $\tau$ be a minimal cone in $\Sigma$. Then for any $\sigma^{\prime} \in \Sigma^{\prime}$, we have

and therefore

$$
\left(\pi^{*} \varphi_{\tau}\right)_{\sigma^{\prime}}= \begin{cases}\varphi_{\tau, \sigma^{\prime}} & \text { if } \sigma^{\prime} \in \Sigma^{\prime} \\ 0 & \text { otherwise }\end{cases}
$$

On the other hand, we have

$$
\pi^{*} \varphi_{\tau}= \begin{cases}\varphi_{\tau} & \text { if } \tau \in \Sigma^{\prime} \\ 0 & \text { otherwise }\end{cases}
$$

$$
\pi^{*} F(\tau)=F(\tau) \cap X^{\prime}= \begin{cases}F(\tau) & \text { if } \tau \in \Sigma^{\prime} \\ 0 & \text { otherwise. }\end{cases}
$$

Our assertion follows, because the ring $R_{\Sigma} / M R_{\Sigma}$ is generated by the images of the $\varphi_{\tau}$.

Now consider the case where $X$ and $X^{\prime}$ are complete. Then we can identify the pull-back map $\pi^{*}: A^{*}(X) \rightarrow A^{*}\left(X^{\prime}\right)$ with $\pi^{*}: H^{*}(X) \rightarrow H^{*}\left(X^{\prime}\right)$. Moreover, the map $R_{\Sigma} / M R_{\Sigma} \rightarrow H^{*}(X)$ identifies with $i^{*}: H^{*}\left(X \times_{T} E T\right) \rightarrow H^{*}(X)$ where $i: X \rightarrow X \times_{T} E T$ is the inclusion of $X$ in $X \times_{T} E T$ as an arbitrary fiber of the fibration $X \times_{T} E T \rightarrow B T$. In this case, our assertion follows from functoriality of pull-back in cohomology.

Finally, consider the general case. Then there exist equivariant completions $i: X \rightarrow \bar{X}$ and $i^{\prime}: X^{\prime} \rightarrow \overline{X^{\prime}}$ and an equivariant morphism $\bar{\pi}: \overline{X^{\prime}} \rightarrow \bar{X}$ which extends $\pi$. Therefore, our assertion follows from the preceding discussion.

(ii) By Corollary 1.2, the images of the $\varphi_{\tau^{\prime}}\left(\tau^{\prime} \in \Sigma^{\prime}\right)$ generate the vector space $R_{\Sigma^{\prime}} / M R_{\Sigma^{\prime}}$. Moreover, it follows from 2.3 that

$$
\pi_{*} \varphi_{\tau^{\prime}}= \begin{cases}\varphi_{\tau} & \text { if the restriction of } \pi \text { to } F\left(\tau^{\prime}\right) \text { is finite over its image } \\ 0 & \text { otherwise. }\end{cases}
$$

On the other hand, the same property holds for the $\pi_{*} F\left(\tau^{\prime}\right)$. We conclude by 3.1.

Corollary. For any smooth, complete torus embedding $X$, with fan $\Sigma$, the following diagram commutes:

$$
\begin{array}{ccc}
R_{\Sigma} & \rightarrow & R_{N} \\
\downarrow & & \downarrow \\
A^{*}(X) & \rightarrow & \mathbf{Q}
\end{array}
$$

where $\int_{X}: A^{*}(X) \rightarrow \mathbf{Q}$ is the degree, $\pi_{*}: R_{\Sigma} \rightarrow R_{N}$ is the push-forward for the morphism $\pi: X \rightarrow$ point, and the map $R_{N} \rightarrow \mathbf{Q}$ is evaluation at 0 .

3.3. The Halphen ring of the torus. Given a $d$-dimensional torus $T$, we denote by $\mathrm{Hal}(T)$ the direct limit of the rational Chow rings of all smooth, complete embeddings of $T$. We call $\mathrm{Hal}(T)$ the Halphen ring of $T$. This terminology is due to Casas and Xambó, who introduced and described the Halphen ring of conics; see [9]. Then DeConcini and Procesi defined the Halphen ring (or "ring of conditions") of any spherical homogeneous space, see [10]. 
A recent result of Fulton and Sturmfels asserts that $\mathrm{Hal}(T)$ is isomorphic to the polytope algebra; see [14]. This result can be rederived as follows: by 3.1 , the algebra $\mathrm{Hal}(T)$ is isomorphic to $R / M R$ where $R$ denotes the algebra of piecewise polynomial functions on $N_{\mathbf{Q}}$. On the other hand, $R / M R$ is isomorphic to the polytope algebra by 1.5 .

By work of DeConcini and Procesi (see [10]), the algebra $\mathrm{Hal}(T)$ governs intersection theory on $T$. More precisely, given $n$ cycles $Y_{1}, \ldots, Y_{n}$ in $T$ whose codimensions sum up to $d$, we can define an intersection number $\left(Y_{1} \cdots Y_{n}\right)_{T}$ as the number of common points to translates $t_{1} Y_{1}, \ldots, t_{n} Y_{n}$ for all $\left(t_{1}, \ldots, t_{n}\right)$ in a non-empty open subset of $T^{n}$. This intersection number gives rise to a bi-additive map $Z^{n}(T) \times Z^{d-n}(T) \rightarrow \mathbf{Z}$ where $Z^{n}(T)$ is the free abelian group on cycles of codimension $n$ in $T$. Denote by $C^{n}(T)$ the quotient of $Z^{n}(T)$ by the orthogonal of $Z^{d-n}(T)$ for this pairing. Then the graded vector space $C^{*}(T)=\bigoplus_{n} C^{n}(T) \otimes_{\mathbf{z}} \mathbf{Q}$ is isomorphic to $\operatorname{Hal}(T)$, and this isomorphism is compatible with intersection products. In fact, this result holds for any spherical homogeneous space; see [10] Theorem 6.3 and also [9] for the space of conics.

Theorem. Let $Y_{1}, \ldots, Y_{n}$ be cycles in $T$ such that $\sum_{i=1}^{n} \operatorname{codim}_{T}\left(Y_{i}\right)=d$.

(i) There exists a smooth, complete $T$-embedding $X$ such that the closures of $Y_{1}, \ldots, Y_{n}$ in $X$ have proper intersection with all orbit closures.

(ii) For $X$ as in (i), there exist $T$-stable cycles $Z_{1}, \ldots, Z_{n}$ such that $\left[\overline{Y_{i}}\right]=\left[Z_{i}\right]$ in $A^{*}(X)$ for $1 \leq i \leq n$. Moreover, if $f_{i}$ denotes the equivariant cohomology class of $Z_{i}$ in $H_{T}^{*}(X) \subset$ $R$, then the image of $f_{i} \bmod M R$ depends only on $Y_{i}$.

(iii) For $f_{1}, \ldots, f_{n}$ as in (ii), we have

$$
\left(Y_{1} \cdots Y_{n}\right)_{T}=\pi_{*}\left(f_{1} \cdots f_{n}\right)
$$

where $\pi$ maps $X$ to a point, and $\pi_{*}$ is described in 2.3.

(iv) If moreover $Y_{1}, \ldots, Y_{d}$ are divisors in $T$, with equations $F_{1}, \ldots, F_{d} \in \mathbf{C}[T]$ and Newton polytopes $\mathcal{N}_{1}, \ldots, \mathcal{N}_{d}$, then we can take for $f_{i}$ the opposite of the support function of $\mathcal{N}_{i}$, and we have

$$
\left(Y_{1} \cdots Y_{d}\right)_{T}=d ! V\left(\mathcal{N}_{1}, \ldots, \mathcal{N}_{d}\right)
$$

where $V$ denotes the mixed volume.

Observe that the functions $F_{1}, \ldots, F_{d}$ in (iv) are only defined up to multiplicative units in $\mathbf{C}[T]$. Therefore, the Newton polytopes are defined up to translation by elements of $M$. But this does not change the image of $f_{i}$ in $R / M R$, neither the mixed volume of $\mathcal{N}_{1}, \ldots, \mathcal{N}_{d}$

Proof. (i) is a special case of [10] Theorem 4.7.

(ii) The existence of $Z_{i}$ follows from [15]. Moreover, by 3.1, the image of $f_{i}$ in $R / M R$ identifies with the class of $Z_{i}$ in $\operatorname{Hal}(T)$, i.e. with the class of $\overline{Y_{i}}$.

(iii) By [10] Theorem 6.3, we have

$$
\left(Y_{1} \cdots Y_{n}\right)_{T}=\int_{X}\left[\overline{Y_{1}}\right] \cdots\left[\overline{Y_{n}}\right]
$$

and hence

$$
\left(Y_{1} \cdots Y_{n}\right)_{T}=\int_{X}\left[Z_{1}\right] \cdots\left[Z_{n}\right]
$$


On the other hand, the diagram

$$
\begin{array}{ccc}
H_{T}^{*}(X) & \rightarrow & R_{N} \\
\downarrow & & \downarrow \\
A^{*}(X) & \rightarrow & \mathbf{Q}
\end{array}
$$

commutes by 3.2. Moreover, $\pi_{*}\left(f_{1} \cdots f_{n}\right)$ is a constant function, because $f_{1} \cdots f_{n}$ has degree $d$, and $\pi_{*}$ has degree $-d$. Therefore, we have

$$
\int_{X}\left[Z_{1}\right] \cdots\left[Z_{n}\right]=\pi_{*}\left(f_{1} \cdots f_{n}\right) .
$$

(iv) Choose $X$ as in (i), and denote its fan by $\Sigma$. For any one-dimensional cone $\tau \in \Sigma$, denote by $n_{\tau}$ the generator of the semigroup $\tau \cap N$. Then $F_{i}$ is a rational function on $X$, with divisor

$$
\overline{Y_{i}}+\sum_{\tau} H_{i}\left(n_{\tau}\right) F(\tau)
$$

where $H_{i}$ denotes the support function of $\mathcal{N}_{i}$. So we can take

$$
\left[Z_{i}\right]=-\sum_{\tau} H_{i}\left(n_{\tau}\right) F(\tau)
$$

which amounts to

$$
f_{i}=-\sum_{\tau} H_{i}\left(n_{\tau}\right) \varphi_{\tau}=-H_{i}
$$

Finally, the formula for $\left(Y_{1} \cdots Y_{n}\right)_{T}$ follows from Corollary 2.4.

R e m a r k. Statement (iv) is a variant of a result of Bernstein and Kouchnirenko; see [2], [18] Théorème III' and also [13] 5.5. It can be considered as a generalization of the theorem of Bézout.

\section{Enumerative geometry in spherical homogeneous spaces.}

4.1. The Newton polytope of a divisor in a spherical homogeneous space. Let $G$ be a connected reductive complex algebraic group, and let $H$ be a closed subgroup. The homogeneous space $G / H$ is spherical if it contains a dense orbit for the action of some Borel subgroup of $G$. We will extend Bernstein-Kouchnirenko's theorem to spherical homogeneous spaces. We begin with some notation and results on these spaces; see e.g. [17] for more on this topic.

Let $G / H$ be a spherical homogeneous space. Then we may choose a Borel subgroup $B$ of $G$ such that $B H$ is open in $G$, and such a $B$ is unique up to conjugation by $H$. The dense $B$-orbit in $G / H$ is $B H / H \simeq B / B \cap H$.

We denote by $K$ the field of rational functions on $G / H$, and by $K^{(B)}$ the multiplicative group of eigenvectors of $B$ in $K$. Observe that any invariant of $B$ in $K$ is a constant function. It follows that every $F \in K^{(B)}$ is determined by the associated character $\chi_{F}$ of $B$, up to a scalar. We denote by $M$ the set of all $\chi_{F}$ for $F \in K^{(B)}$; then $M$ is a free abelian group of finite rank $d$. The integer $d$ is called the rank of $G / H$.

We denote by $\mathcal{V}$ the set of all $G$-invariant discrete valuations $v: K \rightarrow \mathbf{Q} \cup\{\infty\}$. Restriction to $K^{(B)}$ defines a map $\rho: \mathcal{V} \rightarrow \operatorname{Hom}_{\mathbf{Z}}(M, \mathbf{Q}):=N_{\mathbf{Q}}$. This map is injective, and its image is a cone in $N_{\mathbf{Q}}$; see [17]. We identify $\mathcal{V}$ with its image, and we call it the valuation cone.

We denote by $\mathcal{D}$ the set of all irreducible, $B$-stable divisors in $G / H$. Then $\mathcal{D}$ is a finite set; any $D \in \mathcal{D}$ defines a $B$-invariant normalized, discrete valuation $v_{D}$ of $K$. To 
any $D \in \mathcal{D}$ we associate a character $\chi_{D}$ of $B$, which is determined up to translation by a character of $G$. For this, we assume that the $\operatorname{ring}$ of regular functions on $G$ is a UFD (this assumption can always be fulfilled if we replace $G$ by a finite cover $\tilde{G}$, and $H$ by its preimage in $\tilde{G}$ ). Then the inverse image of $D$ in $G$ is an effective divisor, which is stable by left multiplication by $B$. Hence any equation $F_{D}$ of this divisor is an eigenvector of $B$, and we take for $\chi_{D}$ the weight of $F_{D}$. If $F_{1}, F_{2}$ are two such equations, then $F_{2} F_{1}^{-1}:=\varphi$ is a regular, nowhere vanishing function on $G$, and hence $\varphi$ is a scalar multiple of a character of $G$. This explains the indeterminacy of $\chi_{D}$. For any $F \in K^{(B)}$, we have $\chi_{F}=\sum_{D \in \mathcal{D}} v_{D}(F) \chi_{D}$ modulo the character group of $G$.

Let $Y \subset G / H$ be an effective divisor. Translating $Y$ by some $g \in G$, we may assume that no component of $Y$ belongs to $\mathcal{D}$. It means that $Y \cap(B / B \cap H)$ is dense in $Y$. Because the ring of regular functions on $B / B \cap H$ is a UFD, we may choose a generator $F$ of the ideal of $Y \cap(B / B \cap H)$ in this ring. Then $F$ belongs to $K$, and $F$ is determined up to multiplication by an element of $K^{(B)}$ (the group of regular, nowhere vanishing functions on $B / B \cap H)$. We define the Newton polytope of $Y$ by

$$
\mathcal{N}_{Y}=\left\{-\sum_{D \in \mathcal{D}} v_{D}(F) \chi_{D}+m \mid m \in M_{\mathbf{Q}} \text { and } v(m) \geq v(F) \forall v \in \mathcal{V} \cup \mathcal{D}\right\}
$$

Proposition. (i) The set $\mathcal{N}_{Y}$ is a convex polytope in $M_{\mathbf{Q}}$. Moreover, $\mathcal{N}_{Y}$ is uniquely determined up to translation by a character of $G$.

(ii) For any effective divisors $Y^{\prime}, Y^{\prime \prime}$ in $G / H$, we have $\mathcal{N}_{Y^{\prime}+Y^{\prime \prime}}=\mathcal{N}_{Y^{\prime}}+\mathcal{N}_{Y^{\prime \prime}}$.

(iii) In the case where $G$ is a torus and $H$ is the trivial subgroup, $\mathcal{N}_{Y}$ is the usual Newton polytope.

Proof. (i) The set $\mathcal{N}_{Y}$ depends on the choice of $g$ and $F$. We use the notation $\mathcal{N}_{g, F}$ until we have proved the "unicity" of this object.

First observe that the map

$$
\begin{aligned}
& F: \quad \mathcal{V} \rightarrow \mathbf{Q} \\
& v \rightarrow v(F)
\end{aligned}
$$

is convex and piecewise linear. Therefore, the set $\mathcal{N}_{g, F}$ is defined by finitely many linear inequalities. Moreover, $\mathcal{N}_{g, F}$ does not contain any half-line. Otherwise, there would exist $m \in M$ such that $m \neq 0$ and $v(m) \geq 0$ for all $v \in \mathcal{V} \cup \mathcal{D}$. This $m$ would be the weight of a non-constant $F \in K^{(B)}$ with $F$ regular on any equivariant completion of $G / H$, a contradiction. Therefore, $\mathcal{N}_{g, F}$ is a convex polytope.

Denote by $\Omega_{Y}$ the set of all $g \in G$ such that no component of $g Y$ belongs to $\mathcal{D}$. Then $\Omega_{Y}$ is an open subset in $G$, and $\Omega_{Y}$ contains the identity element $1 \in G$. For any $g \in \Omega_{Y}$, an equation of $g Y$ in $B / B \cap H$ is $g F$. Moreover, we have by semi-continuity $v_{D}(g F)=v_{D}(F)$ for all $g \in \Omega_{Y}$. It follows that $\mathcal{N}_{g, g F}=\mathcal{N}_{1, F}$.

Let $F_{1}, F_{2}$ be two generators of the ideal of $Y \cap(B / B \cap H)$. Denote by $\mathcal{N}_{1}$ and $\mathcal{N}_{2}$ the corresponding Newton polytopes. Write $F_{2}=\varphi F_{1}$ for some $\varphi \in K^{(B)}$. Then the character $\chi_{\varphi}-\sum_{D \in \mathcal{D}} v_{D}(\varphi) \chi_{D}$ of $B$, extends to a character of $G$. Now it is easy to check that $\mathcal{N}_{1}$ and $\mathcal{N}_{2}$ differ by translation by $\chi$.

(ii) is a direct verification.

(iii) If $G$ is a torus, then $B$ is equal to $G$, and hence the set $\mathcal{D}$ is empty. Moreover, the valuation cone $\mathcal{V}$ identifies with $N_{\mathbf{Q}}$, and the value of $v \in \mathcal{V}$ at a regular function $F$ on $T$ can be computed as follows. Decompose $F$ as a linear combination of characters $m_{1}, \ldots, m_{r}$ 
of $G$, with non-zero coefficients; then $v(F)$ is the minimum of $v\left(m_{1}\right), \ldots, v\left(m_{r}\right)$. The assertion follows.

4.2. A Bézout theorem for spherical homogeneous spaces. Keep the notation of 4.1 . Denote by $\tilde{M}$ the subgroup generated by the $\chi_{D}$ for all $D \in \mathcal{D}$, and by the character group of $G$; then $\tilde{M}$ contains $M$, and moreover any element of $\tilde{M}$ is the difference of two dominant weights which are in $\tilde{M}$. For such a dominant weight $\lambda$, the dimension of the corresponding simple $G$-module is the value at $\lambda$ of a polynomial function $\Phi$ (Weyl's dimension formula). Denote by $\varphi$ the leading term of $\Phi$. Then it follows from [8] 4.1 that the degree of $\varphi$ is $n-d$ where $n$ denotes the dimension of $G / H$.

For any $d$-dimensional convex polytopes $P_{1}, \ldots, P_{n}$ in $\tilde{M}_{\mathbf{Q}}$, the integral of $\varphi$ over the polytope $t_{1} P_{1}+\cdots+t_{n} P_{n}$ is a polynomial function of the non-negative rational numbers $t_{1}, \ldots, t_{n}$ (this follows e.g. from 2.5). This polynomial is clearly homogeneous of degree $n$; its coefficient over the monomial $t_{1} \cdots t_{n}$ will be denoted by $V_{\varphi}\left(P_{1}, \ldots, P_{n}\right)$. In the case where $G / H$ is a torus, $\varphi$ is the constant function 1 , and $V_{\varphi}$ is the mixed volume.

THEOREM. Notation being as above, for any effective divisors $Y_{1}, \ldots, Y_{n}$ in $G / H$, with Newton polytopes $\mathcal{N}_{1}, \ldots, \mathcal{N}_{n}$, we have

$$
\left(Y_{1} \cdots Y_{n}\right)_{G / H}=n ! V_{\varphi}\left(\mathcal{N}_{1}, \ldots, \mathcal{N}_{n}\right) .
$$

Recall that the symbol $\left(Y_{1} \cdots Y_{n}\right)_{G / H}$ stands for the number of common points to translates $g_{1} Y_{1}, \ldots, g_{n} Y_{n}$ for generic $\left(g_{1}, \ldots, g_{n}\right) \in G^{n}$; see 3.3. In the case where $G / H$ is a connected reductive group $\Gamma$, the result above is due to B. Y. Kazarnovskii by a different method, based on the moment map; see [16]. In this case, we can take $G=\Gamma \times \Gamma$ and $H=\Gamma$ embedded diagonally in $\Gamma \times \Gamma$. Moreover, the groups $M$ and $\tilde{M}$ coincide, and they identify with the character group of a maximal torus of $\Gamma$; then the function $\varphi$ is a scalar multiple of the product of all positive roots.

Proof. Consider an effective divisor $Y$ in $G / H$, its equation $F$ and its Newton polytope $\mathcal{N}$ as in 4.1 . Choose a projective, smooth embedding $X$ of $G / H$ where the closure of $Y$ contains no $G$-orbit (such an $X$ exists by [10] Theorem 4.7). Then we have (as in 3.3)

$$
(Y \cdots Y)_{G / H}=\int_{X}[\bar{Y}]^{n}
$$

Observe that the divisor $\bar{Y}$ is base-point-free. Namely, replacing $G$ by a finite cover, we may assume that the invertible sheaf $\mathcal{O}_{X}(\bar{Y})$ is $G$-linearized. Denote by $\sigma$ the canonical section of $\bar{Y}$; then $\sigma$ generates $\mathcal{O}_{X}(\bar{Y})$ over $X \backslash \bar{Y}$. Therefore, the $G$-translates of $\sigma$ generate $\mathcal{O}_{X}(\bar{Y})$ over $X$, because $\bar{Y}$ contains no $G$-orbit.

We denote by $\left(X_{v}\right)_{v \in \mathcal{V}(X)}$ the set of all irreducible, $G$-stable divisors in $X$; then $\mathcal{V}(X)$ is considered as a subset of $\mathcal{V}$. In the Picard group of $X$, we have $0=\operatorname{div}(F)=\bar{Y}-Z$ where $Z$ denotes the $B$-stable divisor

$$
-\sum_{D \in \mathcal{D}} v_{D}(F) \bar{D}-\sum_{v \in \mathcal{V}(X)} v(F) X_{v}
$$

Moreover, $Z$ is base-point-free, because $\bar{Y}$ is. Therefore, it follows from [8] that

$$
\int_{X} Z^{n}=n ! \int_{\mathcal{N}} \varphi(m) d m .
$$


Namely, when $Z$ is ample, this formula is equivalent to Théorème 4.1 in [8]. When $Z$ is base-point-free, then $Z$ is a limit of ample, $B$-stable divisors $Z_{i}$, and the polytopes associated to $Z_{i}$ converge to $\mathcal{N}_{Y}$. Now our statement follows by polarizing the formula above.

\section{References}

[1] M. F. Atiyah and R. Bott, The moment map and equivariant cohomology, Topology 23 (1984), 1-28.

[2] D. N. Bernstein, The number of roots of a system of equations, Functional Anal. Appl. 9 (1975), 183-185.

[3] E. Bifet, Cohomology, symmetry, and perfection, Publ. Mat. 36 (1992), 407-420.

[4] E. Bifet, C. DeConcini and C. Procesi, Cohomology of regular embeddings, Adv. Math. 82 (1990), 1-34.

[5] L. J. Billera, The algebra of piecewise polynomial functions, Adv. Math. 76 (1989), 170183.

[6] L. J. Billera and L. L. Rose, Modules of piecewise polynomial functions and their freeness, Math. Z. 209 (1992), 485-497.

[7] M. Brion, Points entiers dans les polyèdres convexes, Ann. Scient. École Norm. Sup. (4) 21 (1988), 653-663.

[8] M. Brion, Groupe de Picard et nombres caractéristiques des variétés sphériques, Duke Math. J. 58 (1989), 397-424.

[9] E. Casas-Alvero and S. Xambó-Descamps, The Enumerative Theory of Conics after Halphen, Lecture Notes in Math. 1196, Springer, Berlin, 1986.

[10] C. DeConcini and C. Procesi, Complete symmetric varieties II, in: Algebraic Groups and Related Topics, R. Hotta (ed.), Kinokuniya, Tokyo, 1985, 481-514.

[11] A. Grothendieck and J. Dieudonné, Elements de géométrie algébrique I, Inst. Hautes Études Sci. Publ. Math. 4 (1960).

[12] W. Fulton, Intersection theory, Springer, Berlin, 1984.

[13] W. Fulton, Introduction to toric varieties, Princeton University Press, 1993.

[14] W. Fulton and B. Sturmfels, Intersection theory on toric varieties, preprint 1994.

[15] A. Hirschowitz, L'anneau de Chow équivariant, C. R. Acad. Sci. Paris Série I, 298 (1984), 87-89.

[16] B. Y. Kazarnovskii, Newton polyhedra and the Bézout theorem for matrix-valued functions of finite-dimensional representations, Functional Anal. Appl. 21 (1987), 319-321.

[17] F. Knop, The Luna-Vust theory of spherical embeddings, in: Proceedings of the Hyderabad conference on algebraic groups, S. Ramanan (ed.), Manoj-Prakashan, Madras, 1991, 225250.

[18] A. G. Kouchnirenko, Polyèdres de Newton et nombres de Milnor, Invent. Math. 32 (1976), 1-31.

[19] P. McMullen, The polytope algebra, Adv. Math. 78 (1989), 76-130.

[20] P. McMullen, Valuations and dissections, in: Handbook of convex geometry, Volume B, P. Gruber and J. Wills (eds.), North-Holland, 1993, 933-988.

[21] R. Morelli, A theory of polyhedra, Adv. Math. 97 (1993), 1-73.

[22] R. Morelli, The K-theory of a toric variety, Adv. Math. 100 (1993), 154-182.

[23] C. Procesi and S. Xambó-Descamps, On Halphen's first formula, in: Enumerative algebraic geometry (Copenhagen, 1989), Contemp. Math. 123 (1991), 199-211.

[24] A. V. Pukhlikov and A. G. Khovanskii, Finitely additive measures of virtual polytopes, St. Petersburg Math. J. 4 (1993), 337-356.

[25] R. P. Stanley, Combinatorics and commutative algebra, Birkhäuser, Basel, 1983. 